\title{
Intermodulation Distortion in High Dynamic Range Microwave Fiber-Optic Links with Linearized Modulators
}

\author{
James H. Schaffner, Member, IEEE, and William B. Bridges, Fellow Member, IEEE
}

\begin{abstract}
A number of techniques have recently been described to linearize integrated optic intensity modulators, and thus significantly reduce the two-tone intermodulation distortion. The resulting intermodulation distortion produced by these extended linearity modulators then varies as the input power to the fifth-order or higher. When these modulators are included in a fiber-optic link system, the overall intermodulation product is a combination of third-order and higher order terms. In this paper we determine the dynamic range of a cascaded microwave network consisting of a preamplifier, a high dynamic range fiberoptic link with a highly linear modulator, and a post-amplifier. An expression is found that relates the intermodulation power at the output to the relative suppression from the signal level. As an example, a hypothetical $10 \mathrm{GHz}$ low distortion fiber-optic link that has a dynamic range of $125 \mathrm{~dB}$ in a bandwidth of $1 \mathrm{~Hz}$ is cascaded with various preamplifiers, and it is shown that the dynamic range of the system is reduced by as much as $20 \mathrm{~dB}$, depending upon the third-order intercept of the amplifier.
\end{abstract}

\section{INTRODUCTION}

A variety of techniques to reduce the two-tone third-order intermodulation distortion in integrated optic amplitude modulators have been published recently. These include polarization-mixing [1], dual parallel modulators [2], and modified directional coupler modulators [3]. In all of these modulators the optical signal is essentially split into two paths where the degree of modulation in each path is determined by the RF power split, optical power split, and modulator biasing conditions. It is then possible to suppress the thirdorder distortion by summing the two paths with $180^{\circ}$ of phase difference so that the third-order products cancel. This process eliminates the third-order term in the Taylor series expansion of the modulator transfer curve of output optical power versus the applied electrode voltage, and thus the transfer curve is more linear around the modulator's bias point. As a result the residual two-tone third-order intermodulation products vary as the input power to the fifth-order, and consequently the dynamic range of the fiber-optic link is increased. A continuation of this process would then eliminate the fifthorder term in the Taylor series expansion of the transfer curve and thus the intermodulation products would vary as the seventh-order, and so on.

Manuscript received September 17, 1991; revised April 20, 1992.

J. H. Schaffner is with the Hughes Aircraft Company Research Laboratories, Malibu, CA 90265

W. B. Bridges is with California Institute of Technology, Pasadena, CA 91125.

IEEE Log Number 9205525.
While such extended linear dynamic range modulators have exciting potential applications in CATV and microwave communication systems, the fact remains that these links inevitably need pre- and post-amplification of the signal, and these electronic amplifiers generate their own third-order intermodulation distortion. Thus, it is important for the fiber-optic link designer, when trying to choose the optimum system components, to understand how the intermodulation distortion produced by each of the cascaded network elements contributes to the overall system distortion. Rules for determining the overall dynamic range of cascaded microwave networks where each network has third-order intermodulation distortion are well known [4], [5]. These rules have been applied to determine the dynamic range of analog optical links with conventional Mach-Zehnder interferometric modulators covering frequencies up to $22 \mathrm{GHz}$ [6]. However, in a fiber-optic link containing a linearized modulator the residual third-order intermodulation distortion is now proportional to the input powers to the fifth-order or higher. The overall intermodulation distortion is a now a combination of this higher-order term and third-order components from the amplifiers. Thus the previously derived simple formulas for dynamic range no longer apply. A modification of the method developed by Kanaglekar et al. [5] is presented here that allows one to account for the third-order intermodulation power waves generated by each amplifier and the residual third-order intermodulation power generated by the high dynamic range fiber-optic link. This approach is applied specifically to a system with a high dynamic range fiber-optic link that has intermodulation distortion that varies with the fifth-order of the input power, and a new nonlinear expression is obtained for the overall worst-case dynamic range which is still simple enough to program quickly.

\section{Dynamic Range of the System}

The intermodulation performance of a system is usually specified by the intercept point of the linear extrapolated signal and intermodulation power curves as a function of input power as illustrated in Fig. 1. The $n$th order intercept point $X_{n}(\mathrm{dBm})$ is related to the relative suppression, $R(\mathrm{~dB})$, of the $n$th order intermodulation power and the output signal power, $P_{o}(\mathrm{dBm})$, by [4], [5]

$$
R=(n-1)\left[X_{n}-P_{o}\right] \quad(\mathrm{dBm}) .
$$




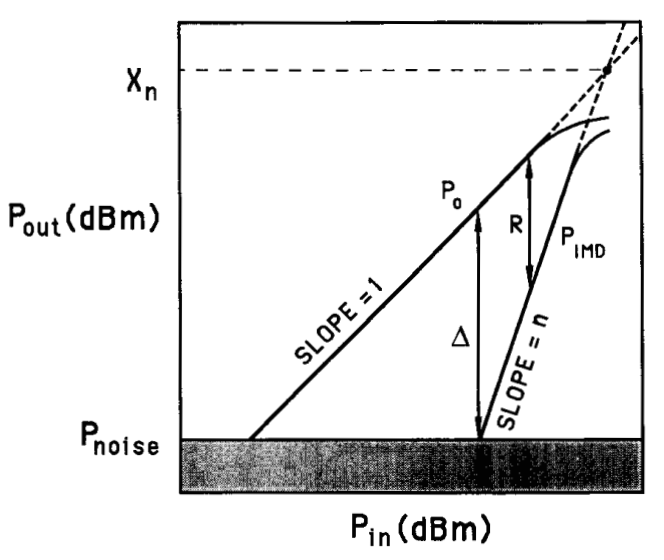

Fig. 1. Output signal power $\left(P_{o}\right)$ and $n$th order intermodulation power $\left(P_{I M D}\right)$ as a function of input power on a $\log -\log$ scale. The $n$th order intercept point, $X_{n}(\mathrm{dBm})$, is the intersection of the extrapolated power curves, and the dynamic range, $\Delta(\mathrm{dB})$, is the difference between the signal power and lowest order intermodulation power (typically third order) when the intermodulation power equals the noise floor in a bandwidth $B$.

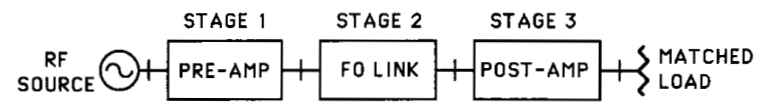

Fig. 2. A high dynamic range fiber-optic link with a preamplifier and a post-amplifier. The amplifiers' intermodulation distortion is third-order and the fiber-optic link's intermodulation distortion is fifth-order.

We have expressed the relationship in decibel units so that its validity is immediately obvious from the quantities in Fig. 1 by simple geometry. We could also express it as

$$
x=r^{1 /(n-1)} p_{o}
$$

where lower case has been used for the same quantities in normal units: $x$ and $p_{o}$ in milliwatts; $r$ is a dimensionless ratio.

When the intermodulation power level is equal to the system noise level, then $R$ is equal to the dynamic range, $\Delta$ :

$$
\Delta=\left(\frac{n-1}{n}\right)\left[X_{n}-P_{\text {noise }}-B\right]
$$

where $P_{\text {noise }}$ is the output noise power $(\mathrm{dBm})$ in a bandwidth of $1 \mathrm{~Hz}$, and $B$ is the bandwidth of the system expressed in decibels.

The cascaded network, depicted in Fig. 2, consists of a first stage preamplifier, a second stage high dynamic range fiber-optic link, a third stage post-amplifier, and a matched termination. The total dynamic range must now be found for the case where the second stage has a fifth-order intercept point (the third-order intermodulation distortion having been canceled by the techniques of [1]-[3]), and the first and third stages have third-order intercept points. The total third-order distortion in the cascaded two-port is found by tracking the intermodulation distortion complex power waves generated by the signal at each two-port by scattering matrix techniques [7]. The relative suppression at the output of a cascaded network has been derived, and for three stages it is [5]

$$
\begin{aligned}
\frac{1}{r_{T}}=\frac{d_{T}}{p_{o}}= & \frac{d_{1}}{p_{1}}+\frac{d_{2}}{p_{2}}+\frac{d_{3}}{p_{3}} \\
& +2 \sqrt{\frac{d_{1} d_{2}}{p_{1} p_{2}}} \cos \left(\theta_{1}-\theta_{2}+\phi_{1}-\phi_{2}\right) \\
& +2 \sqrt{\frac{d_{1} d_{3}}{p_{1} p_{3}}} \cos \left(\theta_{1}-\theta_{3}+\phi_{1}-\phi_{3}\right) \\
& +2 \sqrt{\frac{d_{2} d_{3}}{p_{2} p_{3}}} \cos \left(\theta_{2}-\theta_{3}+\phi_{2}-\phi_{3}\right)
\end{aligned}
$$

where $\sqrt{g_{i}} e^{j \theta_{i}}$ is the complex power wave gain from the output of the $i$ th stage to the load, and $\sqrt{d_{i}} e^{j \phi_{i}}$ is the intermodulation complex power wave generated in the $i$ th stage. The subscript $T$ denotes values for the entire cascade. If $p_{i}$ is the signal power out of the $i$ th stage, then the signal power into the load is $p_{o}=p_{i} g_{i}$ and $r_{i}=p_{i} / d_{i}$ is the relative suppression of the $i$ th stage.

The relationships between the intercept points of the individual stages and their relative suppressions can be found from (2). Explicitly, they are $x_{1}=r_{1}^{1 / 2} p_{1}$ and $x_{3}=r_{3}^{1 / 2} p_{3}$ for the pre- and post-amplifiers with third-order intercept points, and $x_{2}=r_{2}^{1 / 4} p_{2}$ for the low distortion fiber-optic link with a fifth-order intercept point. Therefore, (4) can be written as

$$
\begin{aligned}
\frac{1}{r_{T}}= & \frac{p_{o}^{2}}{x_{1}^{2} g_{1}^{2}}+\frac{p_{o}^{4}}{x_{2}^{4} g_{2}^{4}}+\frac{p_{o}^{2}}{x_{3}^{2}} \\
& +\frac{2 p_{o}^{3}}{x_{1} x_{2}^{2} g_{1} g_{2}^{2}} \cos \left(\theta_{1}-\theta_{2}+\phi_{1}-\phi_{2}\right) \\
& +\frac{2 p_{o}^{2}}{x_{1} x_{3} g_{1}} \cos \left(\theta_{1}+\phi_{1}-\phi_{3}\right) \\
& +\frac{2 p_{o}^{3}}{x_{2}^{2} x_{3} g_{2}^{2}} \cos \left(\theta_{2}+\phi_{2}-\phi_{3}\right) .
\end{aligned}
$$

In (5) $p_{o} / g_{i}$ has been substituted for $p_{i}$, and $g_{3}=1$ with $\theta_{3}=0$ (the gain is unity between the post-amplifier and the load).

The relative suppression $r_{T}$ is a minimum when all of the cosine terms are unity, so that a worst-case equation can be written in terms of $r_{T}$

$$
\begin{aligned}
\frac{1}{d_{T}^{2}}= & \left(\frac{d_{T}^{2}}{x_{2}^{4} g_{2}^{4}}\right) r_{T}^{5}+2 d_{T}\left(\frac{1}{x_{2}^{2} x_{1} g_{2}^{2} g_{1}}+\frac{1}{x_{2}^{2} x_{3} g_{2}^{2}}\right) r_{T}^{4} \\
& +\left(\frac{1}{x_{1}^{2} g_{1}^{2}}+\frac{1}{x_{3}^{2}}+\frac{2}{x_{1} x_{3} g_{1}}\right) r_{T}^{3}
\end{aligned}
$$

where $p_{o}=r_{T} d_{T}$ was used. The minimum dynamic range of the overall system is the value of $r_{T}$ that is determined from (6) by setting $d_{T}=F_{T} k T_{S} g_{T} B$, where $F_{T}$ is the system noise figure, $g_{T}$ is the system gain, $k$ is Boltzmann's constant $(\mathrm{J} / \mathrm{K})$, $T_{S}$ is the system temperature $(\mathrm{K})$, and $B$ is the bandwidth $(\mathrm{Hz})$.

\section{EXAMPLES}

A hypothetical system was designed for operation up to $10 \mathrm{GHz}$ which included a preamplifier and a low distortion fiber-optic link. A program was written that calculated the 
TABLE I

FIBER-OPTIC LINK PARAMETERS

\begin{tabular}{l|l}
\hline Modulator switching voltage, $\mathrm{V}_{\pi}$ & $8 \mathrm{~V}$ \\
Modulator insertion loss & $7.0 \mathrm{~dB}$ \\
Traveling wave electrode impedance & $25 \Omega$ \\
RF power split & $2.4: 1$ \\
Optical power split & $1: 13.8$ \\
Optical power out of the Iaser & $50 \mathrm{~mW}$ \\
Laser RIN & $-160 \mathrm{~dB} / \mathrm{Hz}$ \\
Detector Responsivity & 0.8 \\
Detector Load Resistance & $50 \Omega$ \\
\hline
\end{tabular}

output signal power, two-tone intermodulation power, and noise level of the link as a function of the input RF power. The contribution each stage brings to the overall system performance only depends upon the intercept point, gain, and noise figure of that stage and therefore the low distortion link of stage two could contain any linearized modulator that suppresses the third-order products of the third-order distortion. In the example presented in this section, the fiberoptic link was assumed to include a dual parallel modulator [2] in order to obtain a specific dynamic range for stage two. Modulator and amplifier parameters were taken from commercially available components, and the parameters for the link are listed in Table I. It was found that an RF power split between the dual modulators of $2.4: 1$ and an optical power split of $1: 13.8$ gave an optimum fiber-optic link dynamic range of $125 \mathrm{~dB}$ when the noise power bandwidth was limited to $1 \mathrm{~Hz}$. The link loss was calculated to be $35.7 \mathrm{~dB}$, the fifthorder intercept point was $-2.1 \mathrm{dBm}$, and the noise figure was $51.1 \mathrm{~dB}$. The preamplifier was chosen to be a $6-12 \mathrm{GHz}$ power amplifier with a third-order intercept of $35 \mathrm{dBm}$; it also had $31 \mathrm{~dB}$ of gain and a noise figure of $12 \mathrm{~dB}$.

The dynamic range of the amplifier plus fiber-optic link cascade calculated from (6), was found to be $104.8 \mathrm{~dB}$ in a bandwidth of $1 \mathrm{~Hz}$. Fig. 3(a) shows the output signal power, intermodulation power, and noise level as a function of the input power. The slope of the intermodulation power is 3 $\mathrm{dBm} / \mathrm{dBm}$ until the output power level approaches the intercept point, essentially the slope of the preamplifier distortion. This result is not too surprising because the high fiber-optic link insertion loss means that the amplifier must be driven hard for a specified link output power, thus reducing dynamic range. If the third-order intercept point of the amplifier were increased to $50 \mathrm{dBm}$, then the dynamic range would increase to $114.8 \mathrm{~dB}$, as shown in Fig. $3(\mathrm{~b})$, where the slope of the intermodulation power can now be seen to transition between its two asymptotic values of $3 \mathrm{dBm} / \mathrm{dBm}$ for the amplifier and $5 \mathrm{dBm} / \mathrm{dBm}$ for the fiber-optic link. Fig. 3(c) shows the case of an amplifier with a still higher third-order intercept of 75 $\mathrm{dBm}$ and a resulting dynamic range of $124.8 \mathrm{~dB}$, nearly the value of the fiber-optic link alone. A post-amplifier identical to the preamplifier had little effect on the system dynamic range, lowering it by just $1 \mathrm{~dB}$. The dependence of the dynamic range upon the bandwidth for the asymptotic cases of Fig. 3(a) and (c) can be found in (3). However in the transition region of Fig. 3(b), the slope of the intermodulation distortion (in

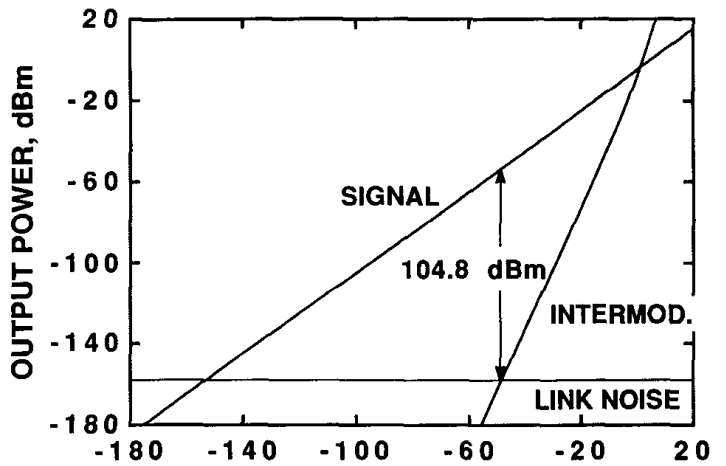

(a)

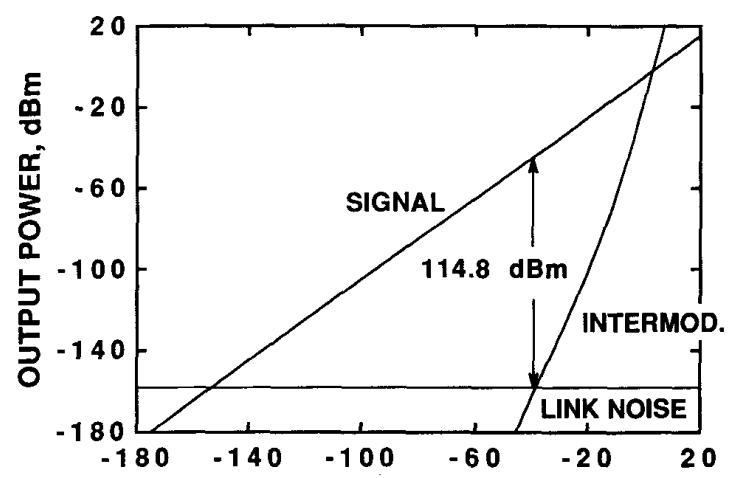

(b)

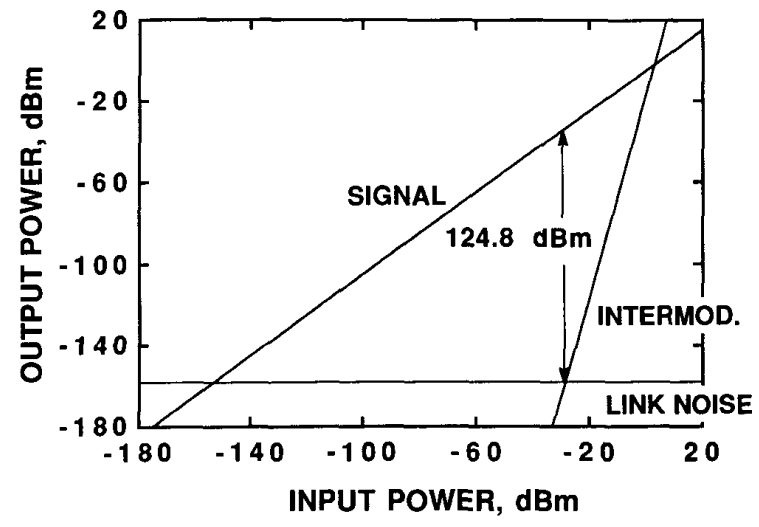

(c)

Fig. 3. The output power of the signal, intermodulation product, and noise as a function of input power for an amplifier-fiber optic link cascade. The fiber optic link has a gain of $-36 \mathrm{~dB}$, a noise figure of $51 \mathrm{~dB}$, and a fifth-order intercept point of $-2 \mathrm{dBm}$. The pre-amplifier gain is $31 \mathrm{~dB}$, the noise figure is $12 \mathrm{~dB}$, and the third-order intercept is (a) $35 \mathrm{dBm}$, (b) $50 \mathrm{dBm}$, and (c) 75 $\mathrm{dBm}$. No post-amplifier was used in these calculations.

decibels) is not constant and the dynamic range does not vary with bandwidth as either $B^{-2 / 3}$ or $B^{-4 / 5}$. In this case (6) must be solved for the dynamic range with the intermodulation distortion equal to the noise level in a bandwidth $\mathbf{B}$ (of course, the dynamic range could be estimated from Fig. 3(b) for different bandwidths by sliding the noise level up by $B$ in decibels). 


\section{DISCUSSION}

An exact expression that relates the dynamic range of a microwave fiber-optic system including a linearized modulator to the network variables of gain, third- and fifth-order intercept points, and noise figure was derived from power wave analysis. In a fiber-optic link, the system dynamic range can be limited by any one of the cascaded stages, or even a combination of all of the stages. Usually, for fiber-optic links containing conventional Mach-Zehnder modulators, the optical link itself is the limiting factor in the dynamic range of the system, however, for a link with a linearized modulator, it is obvious that the amplifiers should play a bigger role in the dynamic range of the system. This was shown in the simple two-stage example presented above where, for that particular example, the relative importance of each stage in determining the overall dynamic range was seen to depend upon the third-order intercept point of the preamplifier. Equation (6) can be used to perform trade-off studies of various network components to determine the optimum design for a high fidelity fiber-optic system.

Furthermore, the analysis presented here is important, not only for the design of high fidelity fiber-optic systems, but also in the experimental determination of the dynamic range of such systems. This is because in conventional networks the thirdorder intercept point can be found from a linear extrapolation of the measured output power and two-tone intermodulation power in decibels; and from (3), the dynamic range can be computed. However, in the high fidelity link, the total intermodulation power may be nonlinear (in decibels) with the input power. Therefore, the dynamic range of the system must be found with the aid of the expressions derived above and the parameters of the individual components. Thus, as linearized modulators begin to impact the fiber-optic industry, the systems into which these modulators are placed can be designed confidently.

\section{ACKNOWLEDGMENT}

The authors would like to thank Dr. G. L. Tangonan for the many helpful technical discussions.

\section{REFERENCES}

[1] L. M. Johnson and H. V. Roussell, "Linearization of an interferometric modulator at microwave frequencies by polarization mixing," IEEE Photon. Technol. Lett., vol. 1, no. 11, pp. 810-811, Nov. 1990.

[2] S. K. Korotky and R. M. De Ridder, "Dual parallel modulation schemes for low-distortion analog optical transmission," IEEE J. Select. Areas Commun., vol. 8, no. 7, pp. 1377-1381, Sept. 1990

[3] M. L. Farwell, Z. Q. Lin, E. Wooten, and W. S. C. Chang, "An electrooptic intensity modulator with improved linearity," IEEE Photon. Technol. Lett., vol. 3, no. 9, pp. 792-795, Sept. 1991

[4] S. E. Wilson, "Evaluate the distortion of modular cascades," $\mathrm{Mi}$ crowaves, pp. 67-70, Mar. 1981.

[5] N. G. Kanaglekar, R. E. Mcintosh, and W. E. Bryant, "Analysis of two-tone, third-order distortion in cascaded two-ports," IEEE Tran. Microwave Theory Tech., vol. 36, no. 4, pp. 701-705, Apr. 1988.
[6] G. E. Betts, L. M. Johnson, and C. H. Cox, III, "High-dynamic-range, low-noise analog optical links using external modulators: Analysis and demonstration," SPIE Proc. High-Frequency Analog Fiber Optic Systems, vol. 1371 (San Jose, CA), Sept. 1990, pp. 252-257.

[7] K. Kurokawa, "Power waves and the scattering matrix," IEEE Trans. Microwave Theory Tech., vol. MTT-13, no. 3, pp. 194-202, Mar. 1965.

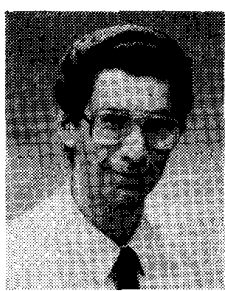

James H. Schaffiner was born in Chicago, IL, on April 27, 1955. He received the B.S., M.S., and Ph.D. degrees from the University of California, Los Angeles, in 1978, 1979, and 1988, respectively. His doctoral research centered on the high frequency characterization of field effect transistors.

From 1978 to 1988 he was a staff member at Hughes Aircraft Company Missile System Group where he worked on antenna arrays and antenna feed systems for microwave and millimeter wave systems. In addition he was involved with the de velopment of microwave and millimeter wave integrated circuits. Since 1988 he has been with the Hughes Research Laboratories. He is now involved with the development of high frequency and high linearity electrooptic components for analog fiber-optic links.

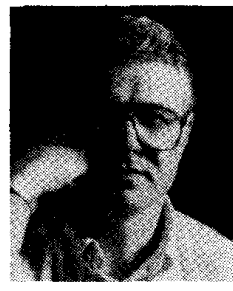

William B. Bridges (S'53-M'61-F'70) was born in Inglewood, CA, in 1934. He received the B.S., M.S., and Ph.D. degrees in electrical engineering from the University of California, Berkeley, in 1956, 1957, and 1962, respectively, and was an Associate in Electrical Engineering from 1957 to 1959, teaching courses in communication and circuits. His graduate research dealt with noise in microwave tubes and electron-stream instabilities (which later became the basis of the Vircator). Summer jobs at RCA and Varian provided stimulating experience with microwave radar systems, ammonia beam masers, and the early development of the ion vacuum pump.

He joined the Hughes Research Laboratories in 1960 as a member of the Technical Staff and was a Senior Scientist from 1968 to 1977, with a brief tour as Manager of the Laser Department in 1969-1970. His research at Hughes involved gas lasers of all types and their application to optical communication, radar, and imaging systems. He is the discoverer of laser oscillation in noble gas ions and spent several years on the engineering development of practical high-power visible and ultraviolet ion lasers for military applications. He joined the faculty of the California Institute of Technology in 1977 as Professor of Electrical Engineering and Applied Physics. He served as Executive Officer for Electrical Engineering from 1979 to 1981. In 1983, he was appointed Carl F Braun Professor of Engineering and conducts research in optical and millimeter wave devices and their applications. Current studies include the millimeter-wave modulation of light and innovation in waveguide gas lasers.

Dr. Bridges is a member of Eta Kappa $\mathrm{Nu}$, Tau Beta Pi, Phi Beta Kappa, and Sigma $\mathrm{Xi}$, receiving Honorable Mention from Eta Kappa Nu as an "Outstanding Young Electrical Engineer" in 1966. He received the Distinguished Teaching Award in 1980 and 1982 from the Associated Students of Caltech, the Arthur L. Schawlow Medal from the Laser Institute of America in 1986, and the IEEE LEOS Quantum Electronics Award in 1988. He is a member of the National Academy of Engineering and the National Academy of Sciences, and a Fellow of the Optical Society of America and the Laser Institute of America. He was a Sherman Fairchild Distinguished Scholar at Caltech in 1974-1975, and a Visiting Professor at Chalmers Technical University, Göteborg, Sweden in 1989. He is coauthor (with C. K. Birdsall) of Electron Dynamics of Diode Regions (New York: Academic Press, 1966.) He has served on various committees of both IEEE and OSA, and was formerly Associate Editor of the IEEE Journal of Quantum Electronics and the Journal of the Optical Society of America. He was the President of the Optical Society of America in 1988, and was a member of the U.S. Air Force Scientific Advisory Board 1985-1989. 